\title{
Keeping Pace with the Expanding Role of Health Coaching
}

\author{
David H. Thom, MD, PhD \\ Division of Primary Care and Population Health, Department of Medicine, Stanford University School of Medicine, Palo Alto, CA, USA.
}

J Gen Intern Med 34(1):5-6

DOI: $10.1007 / \mathrm{s} 11606-018-4730-1$

() Society of General Internal Medicine 2018

$I^{n}$ $\mathrm{n}$ this issue of JGIM, Scuffham et al. report the results of a trial of population-based health coaching to improve disease management. ${ }^{1}$ To better understand these results, it is useful to consider the recent evolution and expansion of the role of health coaching in health care. Health coaching has been defined as a patient-centered process in which a trained coach works with a patient to identify health-related goals, providing education and working with the patient to create action plans to achieve these goals while self-monitoring behaviors to increase accountability. ${ }^{2}$ The conceptual basis for health coaching draws from psychological theories of adult learning and personal development with the aim of creating sustainable behavioral changes based on a patient's personal values. ${ }^{3}$ In practice, health coaching often uses methods derived from models, including Motivational Interviewing, ${ }^{4} \mathrm{~Pa}$ tient Activation, ${ }^{5}$ Health Belief Model, ${ }^{6}$ and Social Cognitive Theory Communication. ${ }^{7}$

The increasing use of health coaching in the delivery of primary care roughly corresponds to the rise of patient-centered models for care of chronic disease, notably the Chronic Care Model, introduced by Wagner over 20 years ago, ${ }^{8}$ which includes patient self-management and decision-making support. The most extensive evidence for the effectiveness of health coaching in primary care comes from randomized controlled trials of health coaching for patients with type 2 diabetes. ${ }^{9,} 10$ Additional studies have found that health coaching can benefit patients with hypertension, obesity, COPD, and heart disease, ${ }^{11,} 12$ though the evidence for these conditions is more limited.

As health coaching has expanded into mainstream health care delivery, it has been adapted and expanded. Inevitably, the definition of health coaching has been stretched and efforts to evaluate the impact of health coaching have not kept pace with the proliferation of models being employed. Scaling up health coaching to large populations of patients through health care delivery systems is an exciting adaptation of the health coaching model, in that it provides an opportunity to reach large numbers of patients. Thus, the randomized controlled trial of population-based health coaching reported by Scuffham et al. is a welcome addition to the literature.

Published online November 7, 2018
In the Costs to Australian Private Insurance - Coaching Health (CAPICHe) trial, Scuffham et al. ${ }^{1}$ randomized 44,418 adults, out of nearly 4 million Australians receiving private health insurance through Bupa Australia, to be actively offer health coaching in addition to usual disease management support from Bupa Health Dialog, versus usual support alone. Patients were eligible for randomization if they had a diagnosis of one or more chronic conditions (heart failure, chronic obstructive pulmonary disease (COPD), coronary artery disease, diabetes, or low back pain) and were predicted to have high claims costs in the next 12 months. Patients with end-stage renal disease, with human immunodeficiency virus infection, under cancer treatment, or having had an organ transplant in the past year were excluded. While patients randomized to the control group could also request health coaching, only $153(1.7 \%)$ did so. Although the paper does not provide a detail description of the training of health coaches or the content of the health coaching, the investigators state that the program was modeled closely on the intervention used by Wennberg et al. ${ }^{13}$ which clearly meets common criteria for health coaching. The primary outcome of the Scuffham study was the dollar amount paid by Bupa Australia for claims from "impactable" hospital admissions over 12 months. Costs paid by the Australian government were not included. Hospital admission not considered impactable, such as maternity care, mental health issues, and same-day diagnostic or treatment procedures (listed in eTable 1), were excluded from the analysis. Secondary outcome was the cost of overnight and same-day admissions analyzed separately. The investigators found only a small (\$66 per member per year) and non-significant difference in total cost, even though the intervention group had significantly lower costs for same-day admissions $(-\$ 40$ per member per year, $p<0.001)$ and fewer such admissions (530 vs 614 per 1000 person-years, $p<0.01$ ). Among patients with diabetes, those in the intervention group incurred even lower same-day admission costs ( $-\$ 86$ per patient per year, $p<0.001)$ and had significant fewer total (1146 vs 1299 per 1000 patient-years, $p=$ 0.008 ) and same-day (612 vs 758 per 1000 patient-years, $p=$ 0.007) admissions.

The CAPICHe trial was modeled on an earlier study by Wennberg ${ }^{14}$ and the authors state that they expected to see a larger effect in the CAPICHe trial, based on the older age of the CAPICHe population (mean age of 72 years vs 37 years for Wennberg). In fact, the opposite seemed to be true, with no effect being seen in patients age 65 and older in the CAPICHe trial, which mirrors the finding of the Wennberg study which found only a small $(3.7 \%)$, non-significant difference in 
hospital admissions for patients 65 and older in contrast to a significant difference of $10.1 \%$ overall. ${ }^{13}$ The CAPICHe study did not measure costs of the health coaching program, but in the paper by Wennberg, the cost of this intervention was estimated to be $\$ 200$ per patient per month.

It is worth noting that, in contrast to non-population-based randomized controlled trials of health coaching, both the current study and the study by Wennberg randomized patients who had not chosen to enroll in a study of health coaching and who, therefore, would be expected to be less receptive to health coaching, on average. Nevertheless, in both studies, approximately $40 \%$ of patients who were contacted with an offer of health coaching accepted at least one coaching session. In contrast, less than $2 \%$ of patients who were simply informed of the availability of coaching actually sought coaching, demonstrating the importance of active outreach for population-based health coaching.

What do we know and what do we need to know about delivering health coaching at the population level? We know that it is possible to do so, though it requires active outreach to patients. We have evidence that the original health coaching model, which is based on a sustained, usually in-person, relationship with a relatively small number of selected patients interested in engaging in health coaching, can also have an impact when scaled up to being offered by telephone to a broad population of patients with multiple conditions who have not been pre-selected for their interest in health coaching. Since health coaching was not originally designed to reduce health care utilization and costs, any evidence for such a reduction seems remarkable. We do not know from either the current study or the study by Wennberg if population-based health coaching improves disease-specific outcomes, patientcentered outcomes, or processes of care. Other studies of population-based health coaching for patients with chronic disease have shown a modest positive impact on quality of life, particularly for patients with diabetes. ${ }^{15-17}$

We also have more to learn about which groups of patients are more likely to benefit from coaching. The strongest evidence for benefits of health coaching comes from randomized controlled trials of patients with diabetes. Like the Scuffham study, the study by Wennberg found that health coaching resulted in a greater reduction in hospital admission for patients with diabetes. In addition, based on the both the Wennberg and Scuffham studies, it appears that population-based health coaching may be most effective at reducing health care utilization and medical costs for patients less than 65 years of age.

The study by Scuffham et al. provides useful information for systems considering implementation of health coaching models. Ultimately, the reach of health coaching will depend on our ability to tailor it to patient needs, using the resources available, under a compelling business model. While shortterm direct health care cost and return on investment is one aspect of a business model, other factors, including improved processes of care, disease control, patient quality of life, and patient and provider satisfaction, are also important considerations. Future studies of population-based health coach are critical to expanding the evidence base for health coaching in the real world of health care delivery.

Corresponding Author: David H. Thom, MD, PhD; Division of Primary Care and Population Health, Department of MedicineStanford University School of Medicine, Palo Alto, CA, USA (e-mail:dthom@stanford.edu).

\section{Compliance with Ethical Standards:}

Conflict of Interest: The author declares that he does not have a conflict of interest.

\section{REFERENCES}

1. Scuffham PA, Byrnes JM, Pollicino C, Cross D, Goldstein S, Ng SK. The impact of population-based disease management services on health care utilisation and costs: results of the CAPICHe trial. J Gen Intern Med. 2018. https://doi.org/10.1007/s11606-018-4682-5.

2. Wolever RQ, Simmons LA, Sforzo GA, Dill D, Kaye M, Bechard EM, Southard ME, Kennedy M, Vosloo J, Yang N. A systematic review of the literature on health and wellness coaching: defining a key behavioral intervention in healthcare. Glob Adv Health Med. 2013;2:38-57.

3. Simmons LA, Wolever RQ. Integrative health coaching and motivational interviewing: synergistic approaches to behavior change in healthcare. Glob Adv Health Med. 2013;2:28-35.

4. Miller WR, Rose GS. Toward a theory of motivational interviewing. Am Psychol. 2009;64:527-37.

5. Hibbard JH, Stockard J, Mahoney ER, Tusler M. Development of the Patient Activation Measure (PAM): conceptualizing and measuring activation in patients and consumers. Health Serv Res. 2004;39:1005-26.

6. Janz NK, Becker MH. The health belief model: a decade later. Health Educ Behav. 1984;11:1-47.

7. Bandura A. Health promotion by social cognitive means. Health Educ Behav. 2004;31:143-64.

8. Wagner EH, Austin BT, Von Korff M. Organizing care for patients with chronic illness. Milbank Q. 1996;74:511-44.

9. Sherifali D, Viscardi V, Bai JW, Ali RM. Evaluating the effect of a diabetes health coach in individuals with Type 2 diabetes. Can $\mathrm{J}$ Diabetes. 2016;40:84-94.

10. Willard-Grace R, Thom DH, Hessler D, Bodenheimer T, Chen EH. Health coaching to improve control of diabetes, hypertension and hyperlipidemia for low-income patients: a randomized controlled trial. Ann Fam Med. 2015;28(1):38-45.

11. Kivelä K, Elo S, Kyngäs $\mathbf{H}, \mathbf{K a ̈ a ̈ r i a ̈ i n e n ~} \mathbf{M}$. The effects of health coaching on adult patients with chronic diseases: a systematic review. Patient Educ Couns. 2014;97:147-57.

12. Benzo R, Vickers K, Novotny PJ, et al. Health Coaching and COPD Rehospitalization: a Randomized Study. Am J Respir Crit Care Med. 2016; 194:672-80.

13. Supplement to Wennberg DE, Marr A, Lang L, O'Malley S, Bennett G. A randomized trial of a telephone care management strategy. Available at https://www-nejm-org.ucsf.idm.oclc.org/doi/suppl/10.1056/ NEJMsa0902321/suppl_file/nejmsa0902321_appendix.pdf. Accessed 30 Sept 2018.

14. Wennberg DE, Marr A, Lang L, O'Malley S, Bennett G. Randomized trial of a telephone care-management strategy. N Engl J Med. 2010; 363: 1245-55.

15. Oksman E, Linna M, Hörhammer I, Lammintakanen J, Talja M. Costeffectiveness analysis for a tele-based health coaching program for chronic disease in primary care. BMC Health Serv Res. 2017; 17:138.

16. Panagioti M, Reeves D, Meacock R, et al. Is telephone health coaching a useful population health strategy for supporting older people with multimorbidity? An evaluation of reach, effectiveness and costeffectiveness using a 'trial within a cohort'. BMC Med. 2018;16:80.

17. Teljeur C, Moran PS, Walshe S, et al. Economic evaluation of chronic disease self-management for people with diabetes: a systematic review. Diabet Med. 2017;34:1040-9. 